\title{
Activation of STAT3 is a key event in TLR4 signaling-mediated melanoma progression
}

\author{
Xiu-Qiong Fu, ${ }^{1,2,3}$, Bin Liu', Ya-Ping Wang ${ }^{1,2}$, Jun-Kui Li ${ }^{1,2}$, Pei-Li Zhu ${ }^{1,2,3}$, Ting Li ${ }^{1}$, Kai-Wing Tse ${ }^{1}$, Ji-Yao Chou ${ }^{1,2}$, \\ Cheng-Le Yin ${ }^{1,2}$, Jing-Xuan Bai ${ }^{1,2}, Y u-X i$ Liu ${ }^{1,2}$, Ying-Jie Chen ${ }^{1,2}$ and Zhi-Ling Yu $u^{1,2,3,4}$
}

\begin{abstract}
Malignant melanoma is aggressive and has a high mortality rate. Toll-like receptor 4 (TLR4) has been linked to melanoma growth, angiogenesis and metastasis. However, signal transduction mediated by TLR4 for driving melanoma progression is not fully understood. Signal transducer and activator of transcription 3 (STAT3) has been identified as a major oncogene in melanoma progression. We found: that TLR4 expression positively correlates with activation/phosphorylation of STAT3 in human melanoma samples; that TLR4 ligands activate STAT3 through MYD88 and TRIF in melanoma cells; and that intratumoral activation of TLR4 increases STAT3 activation in the tumor and promotes tumor growth, angiogenesis, epithelial-mesenchymal transition (EMT) and the formation of an immunosuppressive tumor microenvironment in mice. Further, we found that the effects mediated by activating TLR4 are weakened by suppressing STAT3 function with a dominant negative STAT3 variant in melanoma. Collectively, our work identifies STAT3 activation as a key event in TLR4 signaling-mediated melanoma progression, shedding new light on the pathophysiology of melanoma.
\end{abstract}

\section{Introduction}

Melanoma originated from neural crest-derived melanocytes is an aggressive cancer, with rapid deterioration and high fatality ${ }^{1}$. The pathogenesis of melanoma is complex and is not fully elucidated to date. Although current targeted therapies and immunotherapies for unresectable melanoma are showing exciting clinical results, this disease is still incurable. Understanding the mechanisms of melanoma progression should significantly advance the development of novel therapies for combatting it.

Toll-like receptor 4 (TLR4) is a signaling molecule responsible for clearing pathogens ${ }^{2}$. It is also linked to the development of multiple cancers including melanoma ${ }^{3}$.

\footnotetext{
Correspondence: Zhi-Ling Yu (zlyu@hkbu.edu.hk)

${ }^{1}$ Center for Cancer and Inflammation Research, School of Chinese Medicine, Hong Kong Baptist University, Hong Kong, China

${ }^{2}$ Consun Chinese Medicines Research Centre for Renal Diseases, Hong Kong Baptist University, Hong Kong, China

Full list of author information is available at the end of the article

These authors contributed equally: Xiu-Qiong Fu, Bin Liu

Edited by Q. Chen
}

TLR4 is expressed in $90 \%$ of human primary melanoma lesions and $93 \%$ of metastatic lesions ${ }^{4}$. In mice, TLR4 has been demonstrated to be an active contributor to UVinduced melanomagenesis ${ }^{5}$. Lipopolysaccharide (LPS), a TLR4 ligand, can increase the proliferation and migration of TLR4-postive melanoma cells but not of TLR4-negative ones ${ }^{4}$. LPS has also been shown to promote lung metastasis of melanoma in mice ${ }^{6}$. These facts together substantiate that TLR4 signaling promotes melanoma development. However, mechanisms underlying TLR4 signalingmediated melanomagenesis are not fully understood.

Signal transducer and activator of transcription 3 (STAT3), a transcription factor, is constitutively activated in melanoma ${ }^{7}$. Activation/phosphorylation of STAT3 leads to the transcription of a panel of genes involved in melanoma growth, angiogenesis, metastasis and immune evasion $^{7}$. In tumor tissues from colorectal cancer (CRC) patients, it has been found that expressions of TLR4, MyD88 and STAT3 are positively correlated ${ }^{8}$, although whether a pathway involving the three molecules exists and whether it plays a pathogenic role in CRC are not 
clear. In melanoma, interplay between TLR4 and STAT3 has never been reported.

In this article, we describe four significant findings of our work. First, we found that TLR4 expression and STAT3 activation are positively correlated in human melanoma tissues. Second, we found that TLR4 ligands activate STAT3 through MYD88 (myeloid differentiation primary response gene 88 ) and TRIF (TIR-domain-containing adapter-inducing interferon- $\beta$ ) in melanoma cells and promote STAT3-mediated cellular events in melanoma progression. Third, we found that constitutive TLR4 signaling enhances STAT3 activation in melanoma tissues and promotes tumor growth, angiogenesis and epithelial-mesenchymal transition (EMT) in mice. Forth, we found that overexpression of a dominant negative STAT3 variant in melanoma cells reverses LPS-provoked tumor growth, angiogenesis and EMT and reprograms melanoma microenvironment in mice. Overall, this study demonstrates that activation of STAT3 is a critical event in TLR4 signaling-mediated melanoma progression.

\section{Methods}

\section{Human melanoma tissue microarray}

Human melanoma tissue microarray (malignant melanoma with skin tissue array, 208 cores, Cat. No. ME2082c) was purchased from US Biomax (MD, USA). Information about age, sex and tumor stages of donors is available on the website https://www.biomax.us/ME2082c. The ethics for collecting human tissue samples and the informed consent of patients provided by the company are available in the supplementary documents. Immunohistochemistry (IHC) staining assays using anti-human TLR4 antibody (SCBT: sc-293072; CA, USA) and anti-human phosphoSTAT3 (Y705) antibody (CST: \#9145; MA, USA) were performed following standard protocols provided by Servicebio, Inc. (Wuhan Servicebio Technology Co., Ltd., Hubei, China) and scanned using an automatic digital slide scanner (Pannoramic MIDI II, 3D Histech, Ltd., Budapest, Hungary). The sections were semi-quantified using density quant software in the Quant Center (3D Histech), and protein levels were assessed using the histochemistry score (H-score) system.

\section{Reagents and cell culture}

LPS from Escherichia coli 0111:B4 were purchased from Sigma-Aldrich (St. Louis, USA). Synthetic lipid A from Escherichia coli (MPLAs) was obtained from Invivogen (San Diego, CA, USA). The melanoma cell lines A375 (ATCC ${ }^{\circledR}$ CRL-1619; VA, USA) and B16 (ATCC ${ }^{\circledR}$ CRL6322), and the human umbilical vein endothelial cell (HUVEC) line (ATCC ${ }^{\circledR}$ CRL-1730) were purchased from American Type Culture Collection (ATCC). Human melanoma cell line IGR-1 was purchased from CLS Cell Lines Service (Eppelheim, Germany). All cell lines had been tested for mycoplasma. A375, B16 and IGR1 cells were cultured in DMEM (Gibco, Thermo Fisher, MA, USA), supplemented with $10 \%$ fetal bovine serum (FBS)/ 1\% penicillin-streptomycin (Gibco). The HUVEC cells were cultured in Endothelial Cell Growth Medium (Cell Applications, CA, USA).

To establish stable B16 ${ }^{\mathrm{STAT} 3 \beta}$ and B16 ${ }^{\mathrm{NC}}$ cell lines, B16 cells were transfected with the pCDNA3.1-3xflag-CSTAT3 $\beta$ construct (FitGene Co., Ltd. Guangzhou, China) and the pCDNA3.1-3xflag-C empty vector (FitGene), respectively, and treated with geneticin to select stably transfected clones ${ }^{9}$. STAT3 $\beta$ lacks a 50 bp domain located near the $\mathrm{C}$ terminus compared to the wild-type STAT3. The expression of STAT3 targets Mcl-1, Bcl-xL, VEGF and MMP2 was examined by immunoblotting for confirming the inactivation of STAT3 in the stable B16 ${ }^{\mathrm{STAT} 3 \beta}$ line.

To establish stable $\mathrm{A} 375^{\mathrm{CA}-\mathrm{TLR} 4}$ and $\mathrm{A} 375^{\mathrm{NC}}$ cell lines, A375 cells were transduced with the lentivirus-pLentiGFP-Puro-CMV-CA-TLR4 (lentivirus-CA-TLR4, fused with Myc and Flag tags) and the lentivirus-pLenti-GFPPuro-CMV vector (lentivirus-NC), respectively, and incubated with puromycin for selection of stably transduced clones. The lentivirus-CA-TLR4 and lentivirus-NC were bought from ViGene Biosciences Inc (Shandong, China). The CA-TLR4 fragment was amplified from wide-type TLR4 (NM 138554) lacking DNA sequences encoding the first 20 amino acids (the signal peptide) at $\mathrm{N}$-terminal from a pENTER-TLR4 construct (ViGene Biosciences, CA, USA). TLR4 mRNA level (Fig. S1A) and Myc protein level (Fig. S1B) by RT-qPCR and immunoblotting, respectively, was used to identify the stable lines. The NF$\mathrm{KB}$ and AP-1 transcriptional activities were detected using dual reporter luciferase assays to confirm the activation of TLR4 signaling in the stable A375 ${ }^{\text {CA-TLR4 }}$ line (Fig. S1C).

\section{Immunoblotting}

Cells or tumor tissues were lysed in RIPA lysis buffer containing $50 \mathrm{mM}$ Tris- $\mathrm{HCl}, 1 \% \mathrm{NP}-40,0.35 \%$ sodiumdeoxycholate, $150 \mathrm{mM} \mathrm{NaCl}, 1 \mathrm{mM}$ EDTA (pH7.4), $1 \mathrm{mM}$ phenylmethylsulfonyl fluoride, $1 \mathrm{mM} \mathrm{NaF}, 1 \mathrm{mM} \mathrm{Na}_{3} \mathrm{VO}_{4}$ and $10 \mu \mathrm{g} / \mathrm{mL}$ each of aprotinin, leupeptin and pepstatin A. Protein concentrations were measured using the Quick Start $^{\mathrm{TM}}$ Bradford Protein Assay (Bio-Rad, CA. USA). Primary antibodies used in this study were STAT3 (CST: \#9139), p-STAT3 (Y705; CST: \#9145), TLR4 (SCBT: sc293072), GAPDH (Santa Cruz: sc-32233), Mcl-1 (CST: \# 94296), Bcl-xL (CST: \# 2764), VEGF (Santa Cruz: sc-507) and MMP2 (CST: \# 87809). Immunoblotting was performed following the protocol routinely used in our lab ${ }^{10}$.

\section{Immunofluorescence staining}

A375 cells were treated with LPS $(1 \mu \mathrm{g} / \mathrm{mL})$ for $24 \mathrm{~h}$ and then fixed in $4 \%$ paraformaldehyde (PFA). The fixed cells were permeabilized using $100 \%$ methanol and stained 
with an anti-STAT3 antibody (CST: \#12640; MA. USA) overnight at $4{ }^{\circ} \mathrm{C}$. Cells were then incubated with corresponding secondary antibodies at room temperature for $1 \mathrm{~h}$. Finally, the slices were mounted with DAPIcontaining mounting medium (Abcam) and imaged using a fluorescence microscope (Leica DMI3000 B).

Real-time quantitative polymerase chain reaction analysis

Cells were stimulated with $1 \mu \mathrm{g} / \mathrm{mL}$ of LPS or MPLAs for $48 \mathrm{~h}$. Total RNA of the cells were extracted using Trizol reagent (Thermo Fisher) and reverse transcribed with a reverse transcription kit (Takara, Shiga, Japan) according to manufacturer's protocol. Real-time quantitative polymerase chain reaction (RT-qPCR) was performed using SYBR green reaction mixture (Bio Rad, PA, USA) in the ViiA 7 real-time PCR system (Applied Biosystems). Primer sequences are shown in Table S1. The gene expression data was normalized to the endogenous control GAPDH. The relative expression levels of genes were calculated according to the formula $2^{-\Delta \Delta \mathrm{Ct}}$, where $\Delta \mathrm{Ct}$ is the difference in threshold cycle values between a target and GAPDH, and $\Delta \Delta \mathrm{Ct}=\Delta \mathrm{Ct}$ sample $-\Delta \mathrm{Ct}$ control $^{10}$.

\section{Crystal violet staining}

To investigate proliferative effects of TLR4 ligands, cells were seeded in the $60 \mathrm{~mm}$ dish with $10^{4}$ cells per well and were incubated with LPS $(2 \mu \mathrm{g} / \mathrm{mL})$ or MPLAs $(2 \mu \mathrm{g} / \mathrm{mL})$ for 7 days (medium was refreshed on day 4). To investigate anti-proliferative effects of parthenolide and TAK242 , cells were seeded in the $60 \mathrm{~mm}$ dish with $2 \times 10^{4}$ cells per well and were incubated with parthenolide (1.25, $2.5 \mu \mathrm{g} / \mathrm{mL})$ or TAK-242 $(2.5,5 \mu \mathrm{g} / \mathrm{mL})$ for 7 days (medium was refreshed on day 4). At the end of incubation, cells were fixed using 4\% paraformaldehyde and stained with $0.1 \%$ crystal violet $^{11}$. The culture wells were then washed and photographed. Colony area was measured using Image $(1.74 \mathrm{v})$ software.

\section{Cell invasion assay}

BioCoat $^{\mathrm{TM}}$ Matrigel $^{\circledR}$ Invasion Chambers were obtained from Coring (Cat No: \#354480; NY, USA). LPS $(1 \mu \mathrm{g} /$ $\mathrm{mL})$ - or MPLAs $(1 \mu \mathrm{g} / \mathrm{mL})$-treated cells in $350 \mu \mathrm{L}$ of serum-free DMEM were seeded into the inserts. The lower chambers were filled with $750 \mu \mathrm{L}$ DMEM supplemented with $10 \%$ FBS. After $24 \mathrm{~h}$ of incubation, the cells that did not invade through the matrigel were scraped from the insides of the inserts. Cells on the underside of the matrigel were fixed with $4 \%$ paraformaldehyde and stained with crystal violet. Cells in three microscope areas were photographed and counted ${ }^{10,11}$.

\section{Endothelial cell tube formation assay}

Supernatant collected from A375 cells after $24 \mathrm{~h}$ of LPS $(1 \mu \mathrm{g} / \mathrm{mL})$ or MPLAs $(1 \mu \mathrm{g} / \mathrm{mL})$ stimulation was used as the conditioned media. HUVEC cells were cultured in the conditioned media and plated in 96-well plates coated with $50 \mu \mathrm{L}$ matrigel/well (BD Bioscience, CA, USA) at the concentration $2 \times 10^{4}$ cells/well for $3 \mathrm{~h}$. Tubes in three microscope areas were photographed by microscopy and counted.

\section{Human cytokine array}

Human Cytokine Array Kit was purchased from R\&D Systems (Minneapolis, USA). Conditioned media from A375 cells after $24 \mathrm{~h}$ of MPLAs $(1 \mu \mathrm{g} / \mathrm{mL})$ stimulation were subjected to cytokine array assays following the manufacturer's instructions. The pixel density in each spot of the array was quantified using ImageJ software.

\section{Enzyme-linked immunosorbent assay (ELISA) assay}

Human sICAM-1 (Soluble) Enzyme-linked immunosorbent assay (ELISA) Kit was purchased from ExCell Bio (Shanghai, China). Conditioned media from A375 cells after $24 \mathrm{~h}$ of LPS $(1 \mu \mathrm{g} / \mathrm{mL})$ or MPLAs $(1 \mu \mathrm{g} / \mathrm{mL})$ stimulation were subjected to ELISA assays following the manufacturer's instructions ${ }^{12}$.

\section{Immunohistochemistry staining}

Tumors tissues were fixed in 4\% PFA, dehydrated and embedded in paraffin. The paraffin-embedded tissue blocks were cut into $5 \mu \mathrm{m}$-thick sections and dewaxed, rehydrated, blocked and then incubated with individual primary antibody at room temperature for $60 \mathrm{~min}$. Primary antibodies used in this study were Ki-67 (Abcam: ab16667), CD31 (Abcam: ab9498), E-cadherin (CST: \#3195), N-cadherin (CST: \#13116) and Vimentin (CST: \#5741). Positive signals were developed using diaminobenzidine (DAB) substrate (Dako Carpinteria, CA, USA) under the manufacturer recommended conditions and photographed $^{12}$. Experiment and result assessment were conducted blind.

\section{siRNA transfection}

The TLR4 siRNAs $(238,604,905,1379$, and 1555) were bought from GenePharma (Shanghai, China). The MYD88 siRNA (s9138), TRIF siRNA (s531859) and negative control siRNA (4390843) were bought from ThermoFisher. The sequences of these siRNAs are listed in Table S2. A375 cells were transfected with siRNAs using Lipofectamine RNAiMAX Reagent (ThermoFisher). The gene knockdown efficiency was examined using RTqPCR (Fig. S2).

\section{Luciferase assay}

Dual-Luciferase ${ }^{\circledR}$ Reporter Assay Kit was bought from Promega Corporation (Wisconsin, USA). Cells were seeded in 24-well plates at a density of $5 \times 10^{4}$ cells per well and co-transfected with the STAT3 reporter plasmid 
$4 \times$ M67 pTATA TK-luc $(0.2 \mu \mathrm{g} /$ well, Addgene, USA $)$ and the pRL-CMV vector $(0.1 \mu \mathrm{g} /$ well, promega, USA). After $24 \mathrm{~h}$ of transfection, cells were lysed and subjected to dual reporter luciferase assays according to the manufacturer's instructions. The luminescence of firefly luciferase and the background signal of renilla luciferase were detected using the En Vision Mutilabel Reader (Perkin Elmer, USA $)^{11}$. The transcriptional activity of STAT3 was presented as the luminescence ratio of firefly luc/renilla luc.

\section{Animal experiments}

Male C57/BL6 mice (6-weeks-old) and male $n u / n u$ $\mathrm{BALB} / \mathrm{c}$ mice (6-weeks-old) were bought from the Chinese University of Hong Kong, and maintained in the animal handling room of the Hong Kong Baptist University. All care and handling of animals were performed with the approval of the Research Ethics Committee of Hong Kong Baptist University (Approval number: HASC/15-16/0129). Sample size of each group in our animal studies was calculated based on small-scale studies using the $G^{*}$ power software (Kiel University, version 3.1.9.2). In each calculation, we selected the significant level at $5 \%(\alpha=0.05)$ in a two-tailed test and the power at $90 \%(1-\beta=0.9)$.

To investigate whether constitutive TLR4 signaling promotes melanoma progression, $n u / n u$ BALB/c mice were randomly assigned to two groups $(n=7$; calculated by power analysis based our pilot studies) according to their body weight, and $\mathrm{A} 375^{\mathrm{NC}}\left(1 \times 10^{6}\right.$ cells/mouse $)$ and A375 ${ }^{\text {CA-TLR4 }}\left(1 \times 10^{6}\right.$ cells/mouse $)$ cells were separately subcutaneously (s.c.) injected into the flank of individual mouse. To investigate the role of STAT3 in TLR4 signaling-mediated melanoma progression, C57/BL6 mice were randomly assigned to 4 groups $(n=7$; calculated by power analysis based our pilot studies) according to their body weight, and $\mathrm{B} 16^{\mathrm{NC}}\left(1 \times 10^{6}\right.$ cells/mouse $)$ and B16 $^{\text {STAT3 }}\left(1 \times 10^{6}\right.$ cells/mouse $)$ cells suspended in PBS or LPS were separately s.c. injected into the flank of individual mouse. In the two models, mice were euthanized using $\mathrm{CO}_{2}$ at day 21. Tumors were collected, weighed and sampled for IHC staining, immunoblotting and flow cytometry immunophenotyping. Spleens from the mice bearing $\mathrm{B} 16^{\mathrm{NC}}$ and $\mathrm{B} 16^{\mathrm{STAT} 3 \beta}$ tumors were also collected for immunophenotyping analyses.

To investigate the effects of parthenolide on melanoma tumor growth, B16 $\left(1 \times 10^{6}\right)$ cells were s.c. injected into the flank of individual C57/BL6 mice. At day 7, mice were randomly assigned to four groups $(n=5$; calculated by power analysis based our pilot studies) according to their tumor sizes and intraperitoneally injected with $0.5,1$, $2 \mathrm{mg} / \mathrm{kg} /$ days of parthenolide in $0.2 \mathrm{~mL}$ PBS or with the vehicle ( $0.2 \mathrm{~mL}$ of PBS) for 7 consecutive days. At the end of the experiments, mice were euthanized using $\mathrm{CO}_{2}$. Tumors were collected, weighed and immunoblotted. A tumor with a volume of $<50 \mathrm{~mm}^{3}$ at 7 days after cell injection was excluded from the analysis.

\section{Flow cytometric immunophenotyping}

Spleens and tumors from tumor-bearing mice were used to prepare single cell suspensions. Cells incubated with fluorochrome-conjugated antibodies were detected using flow cytometry (12). The anti-CD3 (553063), antiCD4 (553651), anti-CD8 (551162), anti-NK 1.1 (553164), anti-CD25 (553866), anti-CD86 (553691), anti-CD11c (553802), anti-Gr-1 (561105), anti-CD11b (557397), anti-Foxp3 (563101) and anti-F4/80 (564227) antibodies were bought from BD Biosciences (US). Experiment and data analysis were conducted blind.

\section{Statistical analysis}

Data are presented as mean \pm SD unless otherwise indicated in the figure legends. Statistical analyses were carried out using GraphPad Prism version 5.0 (GraphPad Software, San Diego, CA, USA). Pearson correlation and Spearman coefficient were calculated to assess correlation of TLR4 and phosphorylated STAT3 in the human melanoma tissue microarray. Student's $t$-test was used to compare the difference between two groups. Homogeneity of variance was assessed using Brown-Forsythe test. Comparisons among multiple groups were performed using One-way ANOVA followed by Tukey's test. $P<0.05$ was regarded as statistically significant.

\section{Results}

TLR4 expression and STAT3 phosphorylation are positively correlated in human melanoma samples

To determine the correlation of TLR4 expression and STAT3 activation/phosphorylation, we took advantage of the human melanoma tissue microarray (a total of 208 samples) and performed immunohistochemical staining (Fig. 1a). Protein expression levels were semiquantitatively assessed using the $\mathrm{H}$-score system (Tables S3 and S4). As shown in Fig. 1b, c, protein levels of TLR4 and phosphorylated STAT3 (Y705) were significantly elevated in melanoma tissues compared to that in normal tissues, a finding consistent with previous reports ${ }^{4,7}$. Further analyses showed that TLR4 expression and STAT3 phosphorylation were positively correlated in the 208 samples (Fig. 1d), especially in early-stage $\left(\mathrm{T}_{1} \mathrm{~N}_{0} \mathrm{M}_{0}\right)$ melanomas (samples C4, D9, F2, F9, G9, H8, H9; Fig. 1e). The positive correlation of TLR4 expression and STAT3 phosphorylation was also found in both male and female patient samples (Fig. S3). In different age groups, TLR4 expression and STAT3 phosphorylation were positively correlated in samples from middle-aged (40-60 years old) and elderly ( $>60$ years old) patients but not in samples from young (<40-years-old) patients (Fig. S3). Reasons for the different observations among patients of $<40$ years old and 
a

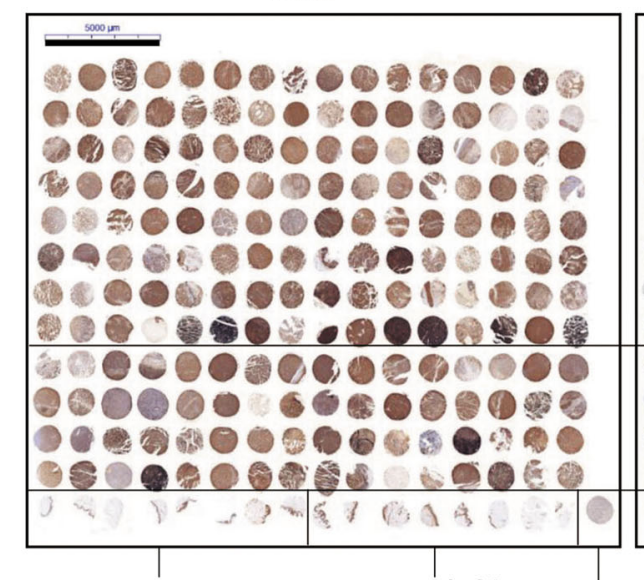

Adjacent normal skin tissue
TLR4

Normal skin Marked point
p-STAT3

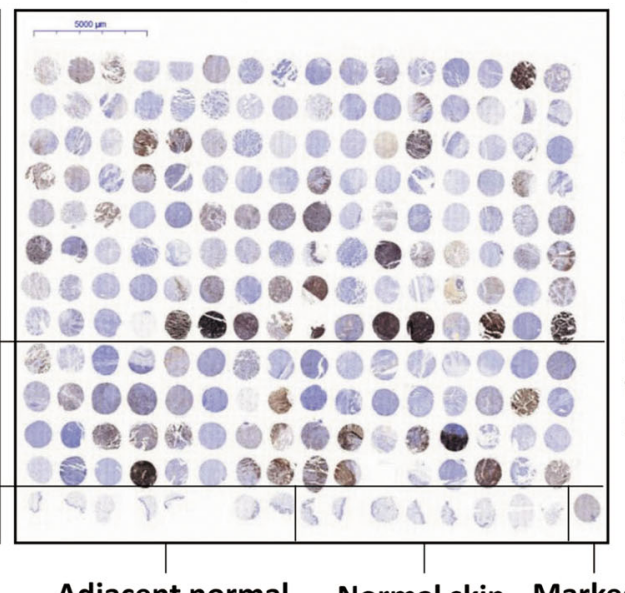

Adjacent normal Normal skin Marked point skin tissue node b
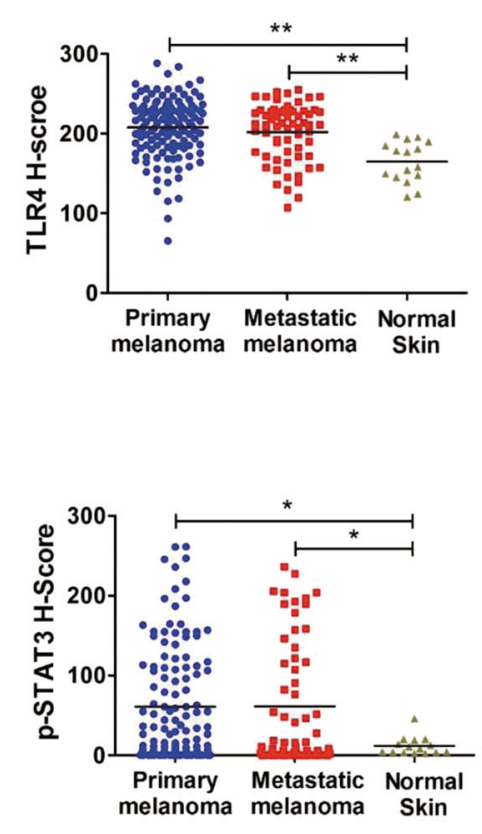

d

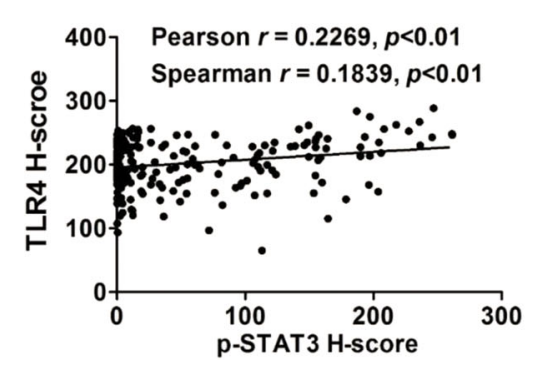

C

TLR4
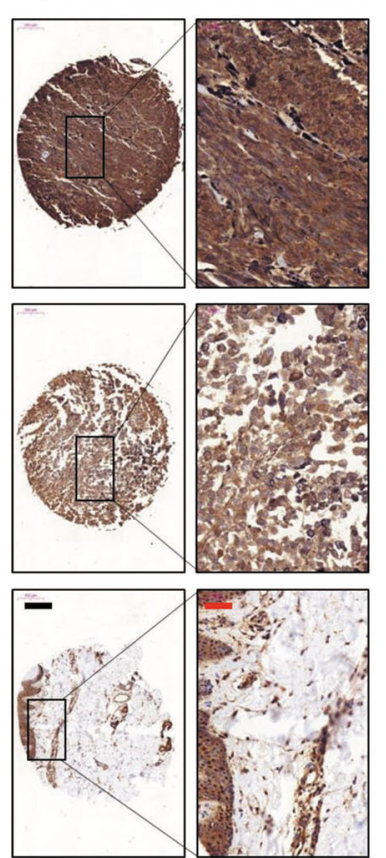

p-STAT3

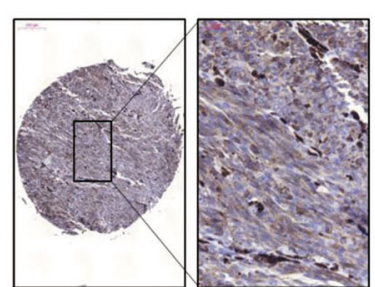

Primary

melanoma

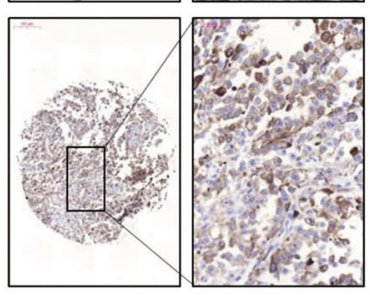

tatic

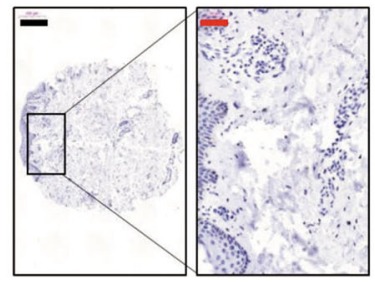

Metastatic melanoma

Normal

skin

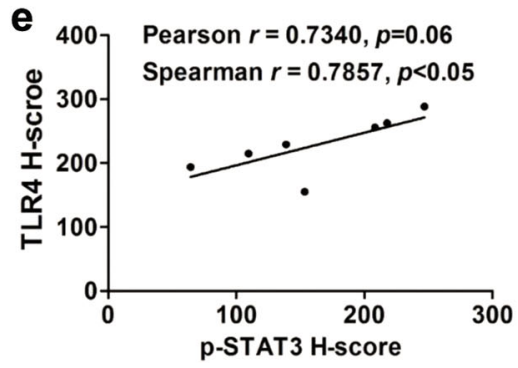

Fig. 1 (See legend on next page.) 
(see figure on previous page)

Fig. 1 TLR4 expression and STAT3 phosphorylation positively correlate in human melanoma samples. a Immunohistochemical (IHC) staining of the expression of TLR4 and phosphorylated STAT3 (p-STAT3; Tyr705) in a human melanoma tissue microarray. Scale bar: $5 \mathrm{~mm}$.

b Quantification of TLR4 and $p$-STAT3 staining intensity in the melanoma tissue microarray. ${ }^{*} p<0.05,{ }^{* *} p<0.01$. $\mathbf{c}$ IHC staining of TLR4 and $p$-STAT3 in representative melanoma specimens and normal skin tissues. Scale bar: black, $200 \mu \mathrm{m}$; red, $50 \mu \mathrm{m}$. d Scatter plots of TLR4 and p-STAT3

immunostaining intensity in human melanoma tissues $(n=208)$. e Scatter plots of TLR4 and p-STAT3 immunostaining intensity in early-stage melanoma tissues $(n=7)$.

$\geq 40$-years-old need to be explored. Collectively, the positive correlation together with the high levels of TLR4 expression and STAT3 phosphorylation in human melanoma tissues suggests a link between TLR4 and STAT3 in melanoma pathogenesis.

\section{TLR4 ligands activate STAT3 through MYD88 and TRIF in melanoma cells}

To determine whether TLR4 signaling involves STAT3 activation in melanoma cells, we used TLR4 ligands to stimulate the cells and examined STAT3 phosphorylation and nuclear localization. Results showed that both LPS and MPLAs, two TLR4 ligands, increased the phosphorylation of STAT3 in multiple melanoma cell lines (Figs. 2a and S4). Once STAT3 was phosphorylated, it translocates into the nucleus where it functions as a transcription factor ${ }^{7}$. We also found that LPS increased STAT3 nuclear localization in A375 cells (Fig. 2b), further confirming the activation of STAT3 by TLR4 ligand stimulation.

Although MPLAs is claimed to specifically activate TLR4 signaling, LPS activates both TLR4 and TLR2 signaling ${ }^{13}$. TLR4 and TLR2 are widely expressed in melanoma cells ${ }^{14}$. To determine the necessity of TLR4 for LPS- and MPLAs-induced STAT3 activation, we knocked down TLR4 in A375 cells using a specific siRNA (siTLR4; 1555) that has been shown to be able to effectively silence TLR $4^{15}$, and then stimulated the cells with TLR4 ligands. Efficient gene knockdown was confirmed by RT-qPCR (Fig. S2). As shown in Fig. 2c, d, LPS and MPLAs significantly increased STAT3 phosphorylation in A375 cells transfected with the negative control (NC) siRNA. Knockdown of TLR4 completely abolished LPS- and MPLAs-enhanced STAT3 phosphorylation. These data verified that the LPS- and MPLAs-induced STAT3 activation is through TLR4 in melanoma.

Upon ligand recognition, TLR4 signals through MYD88-dependent and/or TRIF-dependent pathways ${ }^{16}$. We found that silencing MYD88 or TRIF, like silencing TLR4, abrogated both LPS- and MPLAs-enhanced STAT3 phosphorylation (Fig. 2c, d). MPLAs is reported to specifically trigger TLR4/TRIF signaling ${ }^{17}$. The observation that knockdown of MYD88 abolishes MPLAsenhanced STAT3 phosphorylation suggests that TRIF can be regulated by MYD88 in melanoma. Whether MYD88 directly or indirectly regulates TRIF needs to be further investigated. Nevertheless, the findings indicate that both MYD88 and TRIF are involved in TLR4 ligand-mediated STAT3 activation.

In addition, we found that knockdown of TLR4 lowered the protein level but not the mRNA level of STAT3 in melanoma cells (Figs. 2c, e and S5A, B). STAT3 protein degraded faster in siTLR4-transfected cells than in NC siRNA-transfected cells when protein synthesis was blocked by cycloheximide (CHX) in the cells (Fig. S5C, D). MYD88 or TRIF knockdown did not affect total STAT3 protein levels (Fig. 2c, e). These observations suggest that TLR4 signaling uses different mechanisms to activate STAT3 and to sustain STAT3 protein stability.

\section{TLR4 ligands promote STAT3-mediated cellular events in melanoma progression}

We have observed that TLR4 ligands increase nuclear localization of STAT3 in melanoma cells (Fig. 2b). Here, we examined whether TLR4 ligands affect melanoma progression-related genes that can be transcribed by STAT3 in melanoma cells. RT-qPCR results showed that mRNA levels of BCL2L1 (BCL-XL) and MCL1 (involved in melanoma cell survival $)^{7,18}$, and $M M P-2, M M P-9$ and $V E G F$ (involved in melanoma metastasis and angiogenesis) ${ }^{7,19,20}$ that can be transcriptionally upregulated by STAT3 were elevated upon LPS or MPLAs stimulation in melanoma cells (Fig. 3a). Next, we examined whether TLR4 ligands trigger the above genes-related malignant behaviors in cell models. Results showed that MPLAs and LPS, in a similar manner, promoted proliferation (Fig. 3b) and invasion (Fig. 3c) of melanoma cells. To test whether TLR4 signaling in melanoma cells promotes angiogenesis, conditioned media from MPLAs- and LPS-stimulated A375 cells were used to separately incubate HUVECs, and formed tubes were counted. Results showed that both conditioned media increased tube formation of HUVECs (Fig. 3d), indicating that TLR4 signaling promotes angiogenesis in vitro.

MPLAs has been clinically tested as an immunotherapeutic agent in treating cancers including melanoma (clinicaltrials.gov Identifier: NCT01584115). We have found that MPLAs activates STAT3 in melanoma cells (Fig. 2). Activation of STAT3 is reported to upregulate immunosuppressive cytokines to promote tumor immune evasion ${ }^{7}$. To examine whether MPLAs provokes the 


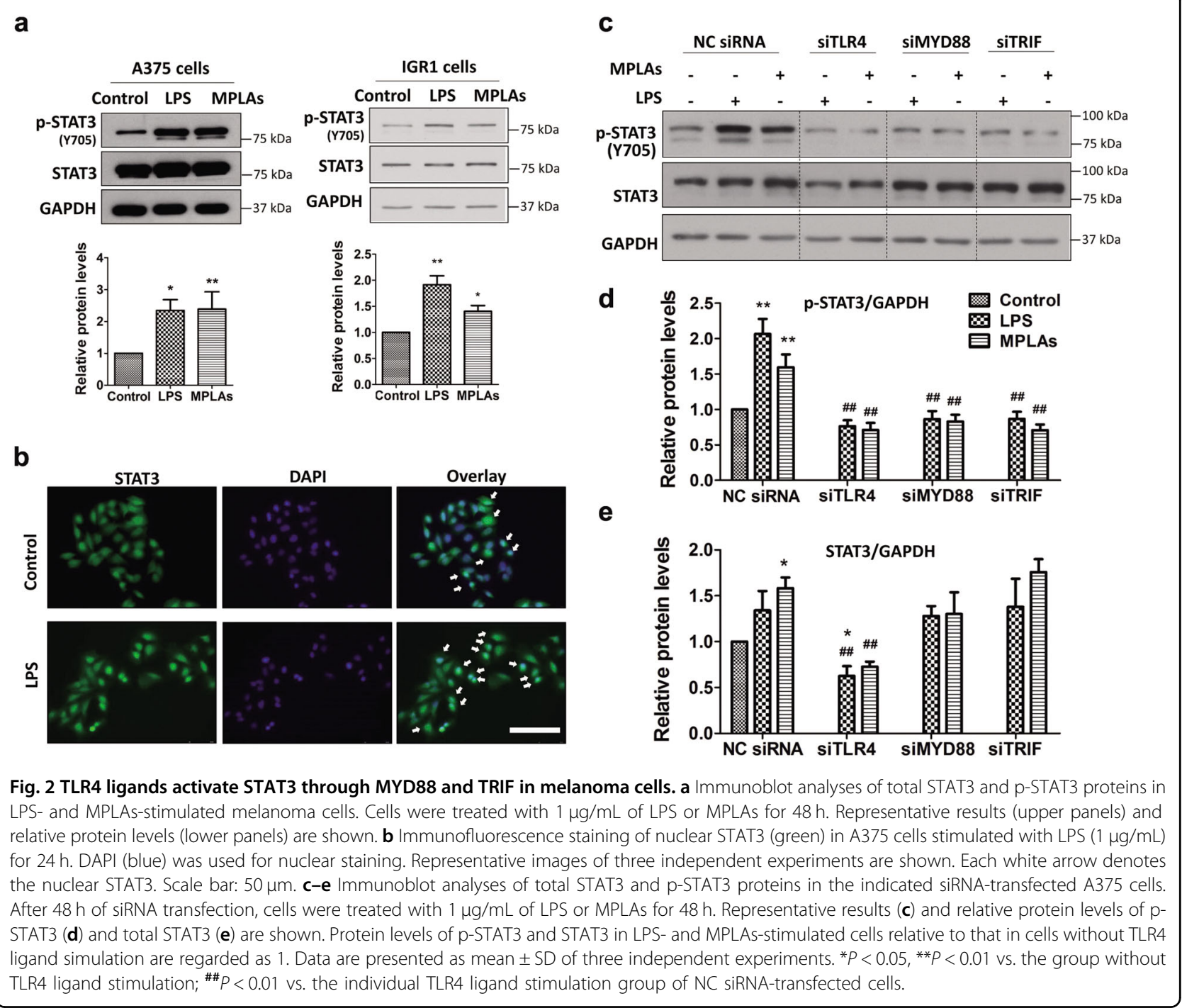

production of immunosuppressive cytokines, we collected the conditioned media from MPLAs-treated A375 cells and conducted a cytokine array assay. Results showed that several immunosuppressive cytokines, including ICAM$1^{21}, \mathrm{IL}_{-}{ }^{22}, \mathrm{CXCL}^{2} 2^{22}, \mathrm{G}-\mathrm{CSF}^{23}$, and PAI- ${ }^{24}$, were increased upon MPLAs stimulation (Fig. 3e, f). G-CSF can be upregulated by TLR4 signaling ${ }^{25}$ and is able to activate STAT3 in melanoma ${ }^{26}$. ICAM-1, IL-6, CXCL12 and PAI1 are transcriptionally regulated by STAT3 ${ }^{27,28}$. ICAM-1 is the most increased in the altered proteins. We quantified it using ELISA assays. Results confirmed that MPLAs significantly increased ICAM-1 secretion; and LPS also increased ICAM-1 secretion (Fig. 3g). Other altered cytokines observed in the cytokine array assay need to be further verified. Notwithstanding, these results show that TLR4 signaling in melanoma cells increases the secretion of immunosuppressive cytokines that can be upregulated by STAT3.
Next, we examined whether activation of STAT3 is required for the cellular events in TLR4 signalingmediated melanoma progression. For this purpose, we established a pair of stable cell lines, B16 ${ }^{\text {STAT3ß }}$ (a line overexpressing STAT3 $\beta$ ) and $\mathrm{B}^{\mathrm{NC}}$ (the negative control cell line harbouring the empty vector). STAT3 $\beta$ is a dominant-negative variant of STAT3, containing the dimerization and DNA-binding domains but without the transactivation domain ${ }^{29}$. Immunoblotting showed that protein levels of STAT3 targets Bcl-xL, Mcl-1, VEGF and MMP2 were lower in B16 ${ }^{\text {STAT3 } \beta}$ cells than in $\mathrm{B} 6^{\mathrm{NC}}$ cells (Fig. 3h), indicating that STAT3 activation is blocked by STAT3 $\beta$. We found that both LPS and MPLAs significantly increased cell proliferation (Fig. 3i) and invasion (Fig. 3j) in $\mathrm{B} 16^{\mathrm{NC}}$ cells but not in B16 ${ }^{\text {STAT3 } \beta}$ cells, suggesting a critical role of STAT3 activation in TLR4 signaling-caused melanoma progression. 


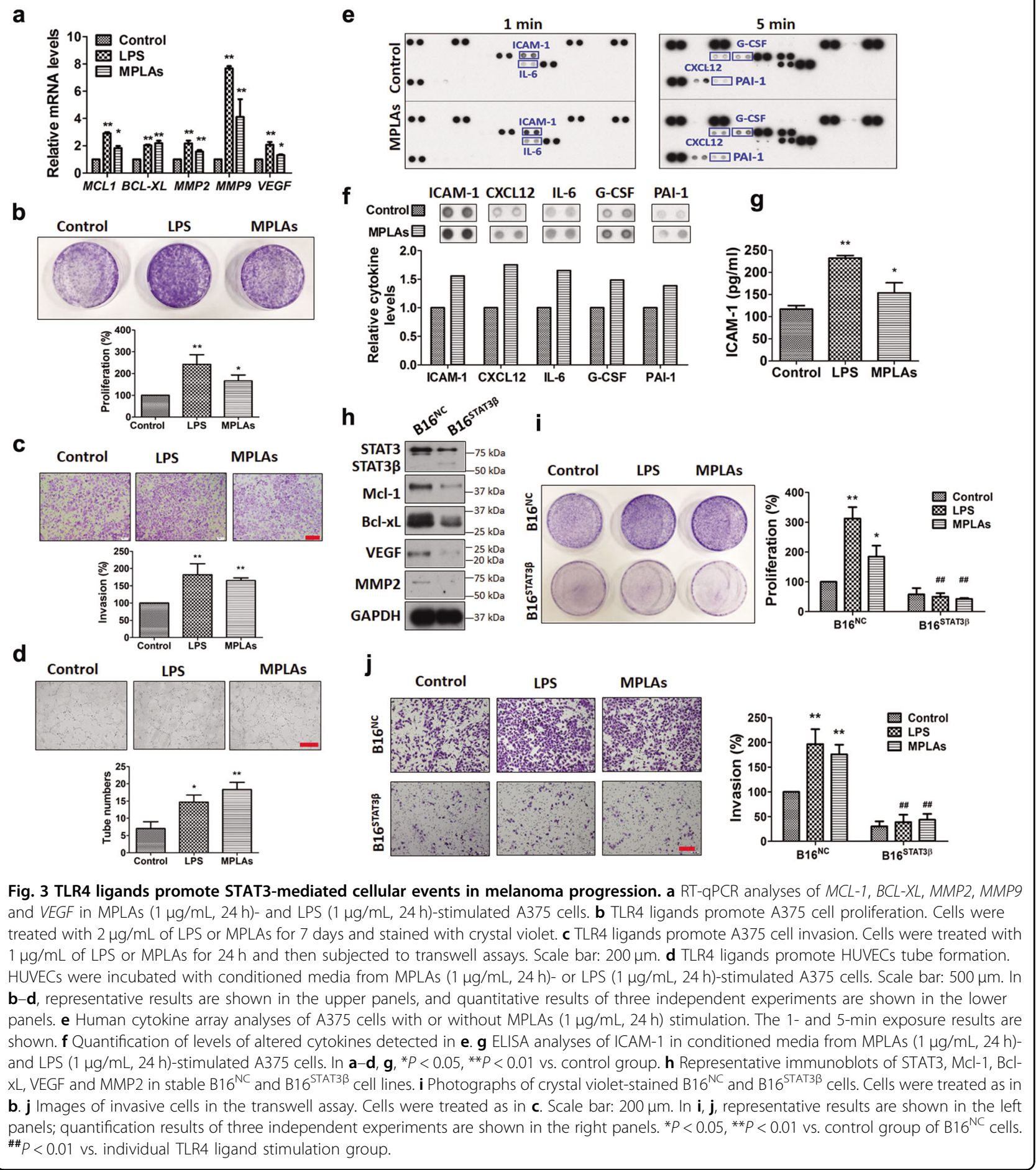

Constitutive TLR4 signaling enhances STAT3 activation and promotes melanoma progression in mice

To determine the relation between TLR4 and STAT3 in melanoma in vivo, we established a pair of stable cell lines, A375 ${ }^{\text {CA-TLR4 }}$ (harbouring a constitutively active variant of TLR4) and $A 375^{\mathrm{NC}}$ (the negative control cell line harbouring the empty vector), then compared STAT3 activity in the two lines, and further compared tumor growth, angiogenesis and EMT in A375 ${ }^{\mathrm{CA}-\mathrm{TLR} 4}$ - and A375 ${ }^{\mathrm{NC}}$ bearing mice. Higher transcriptional activity of two TLR4 downstream transcription factors NF- $\mathrm{kB}$ and AP-1 (Fig. $\mathrm{S} 1 \mathrm{C}$ ) in A375 ${ }^{\mathrm{CA}-\mathrm{TLR} 4}$ cells than in $\mathrm{A} 375^{\mathrm{NC}}$ cells confirmed constitutive TLR4 signaling in A375 ${ }^{\text {CA-TLR4 }}$ cells. It was found that protein level of phosphorylated STAT3 and 
transcriptional activity of STAT3 were higher in A375 ${ }^{\mathrm{CA}-}$ TLR4 cells than in A375 ${ }^{\mathrm{NC}}$ cells (Fig. 4a, b), indicating that constitutive activation of TLR4 enhances STAT3 activation in melanoma cells. EMT is an early event of tumor cell invasion and metastasis ${ }^{30}$. EMT-like morphological features, such as losing cell-cell interaction and a spindleshaped phenotype, were observed in A375 ${ }^{\text {CA-TLR4 }}$ cells (Fig. 4c), suggesting that TLR4 activation promotes EMT in melanoma. In A375 CA-TLR4 and $\mathrm{A} 375^{\mathrm{NC}}$ xenograftsbearing mouse models, weights of A375 ${ }^{\text {CA-TLR4 }}$ xenografts were heavier than those of $\mathrm{A} 375^{\mathrm{NC}}$ xenografts (Fig. 4d). Protein levels of total and phosphorylated STAT3 were upregulated in A375 ${ }^{\mathrm{CA}-\mathrm{TLR} 4}$ tumors (Fig. 4e). IHC staining of tumor tissues showed that protein levels of
Ki-67 (a cell proliferation marker), CD31 (an angiogenic marker), N-cadherin and vimentin (two mesenchymal markers) were increased and E-cadherin (an epithelial marker) was decreased in A375 ${ }^{\text {CA-TLR4 }}$ tumors compared to $\mathrm{A} 375^{\mathrm{NC}}$ tumors (Fig. 4f). Together, these data indicate that activation of TLR4 enhances STAT3 activation in melanoma tissues and promotes tumor growth, angiogenesis and EMT in mice.

\section{Activation of STAT3 is required for TLR4 signaling- mediated melanoma progression in mice}

Next we examined whether activation of STAT3 is required for TLR4 signaling-mediated melanoma progression in vivo. $\mathrm{B} 16^{\mathrm{NC}}$ and $\mathrm{B} 16^{\mathrm{STAT} 3} \beta$ cells suspended a

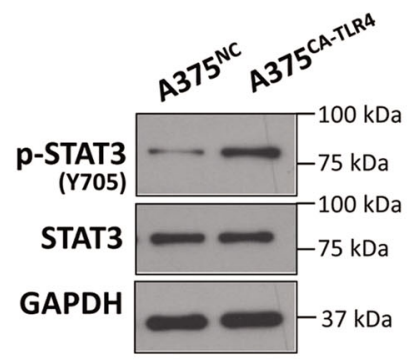

b

STAT3 transcriptional activity

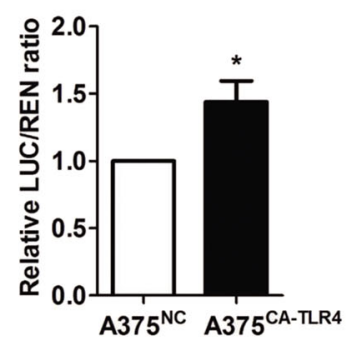

C

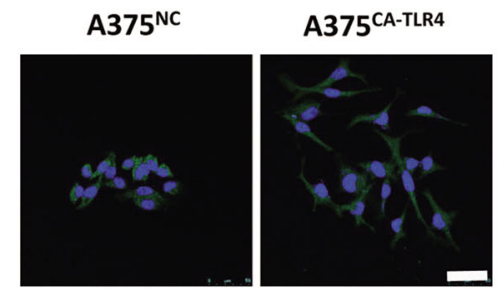

d
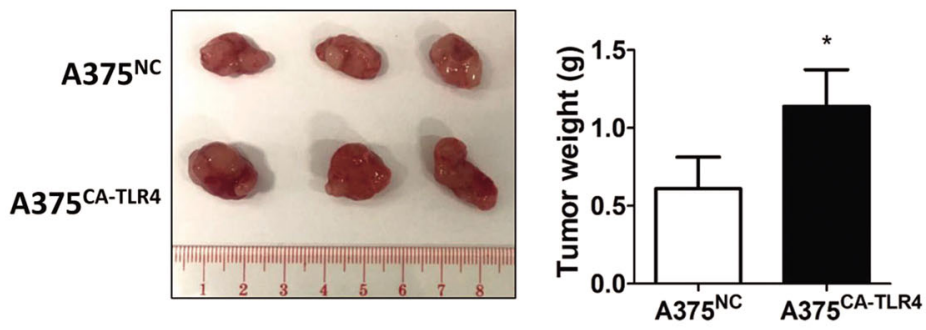

e
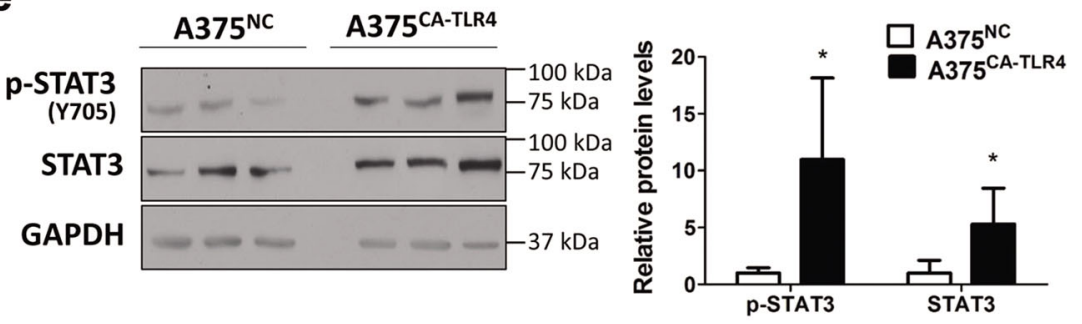

$\mathbf{f}$

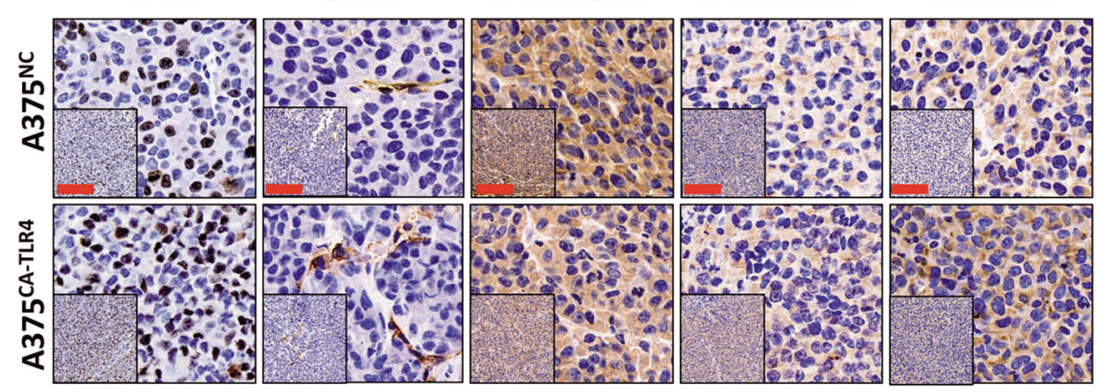

Fig. 4 Constitutive TLR4 signaling enhances STAT3 activation and promotes tumor growth, angiogenesis and EMT in mice. a Representative immunoblots of total and phosphorylated STAT3 in A375 $5^{\mathrm{NC}}$ and A375 $5^{\text {CA-TLR4 }}$ cells. b Transcriptional activity of STAT3 in A375 $5^{\text {NC }}$ and A375 $5^{\text {CA-TLR4 }}$ cells. c Morphological images of A375 ${ }^{\mathrm{NC}}$ and A375 ${ }^{\mathrm{CA}-T L R 4}$ cells. Cells expressing green fluorescent protein (GFP) displayed green fluorescence. DAPI (blue) was used for nuclear staining. Scale bar: $50 \mu \mathrm{m}$. d Representative images (left panel) and weights (right panel) of A375 ${ }^{\mathrm{NC}}$ and A375 ${ }^{\mathrm{CA}-\mathrm{TLR} 4}$ tumors ( $n=7$, two independent experiments). e Immunoblot analyses of total STAT3 and phosphorylated STAT3 (p-STAT3) in A375 ${ }^{\mathrm{NC}}$ and A375 ${ }^{\text {CA-TLR4 }}$ tumors. Representative immunoblotting results are shown in the left panel. Protein levels of p-STAT3 and STAT3 in A375 ${ }^{\text {CA-TLR4 }}$ tumors relative to that in $\mathrm{A} 375^{\mathrm{NC}}$ tumors (regarded as 1) are shown in the right panel. GAPDH was used as a loading control. In $\mathbf{a}$, $\mathbf{c}-\mathbf{e}$, data are shown as the mean \pm SD of three independent experiments. ${ }^{*} P<0.05$ vs. $\mathrm{A} 375^{\mathrm{NC}}$ group. $\mathbf{f}$ Representative $\mathrm{IHC}$ staining results of Ki-67, CD31, E-cadherin, $\mathrm{N}$-cadherin and vimentin in $\mathrm{A} 375^{\mathrm{NC}}$ and $\mathrm{A} 375^{\mathrm{CA}-\mathrm{TLR} 4}$ tumors. Scale bar: $200 \mu \mathrm{m}$. 
separately in PBS in the presence or absence of LPS were subcutaneously injected into the flank of individual C57BL/6 mice. On day 21 after cell injection, mice were euthanized, and tumors were excised and weighed. Similar to previous reports, the $\mathrm{B} 16^{\mathrm{STAT} 3} \mathrm{~B}$ tumors were smaller and lighter than the $\mathrm{B} 16^{\mathrm{NC}}$ tumors (Fig. 5a). We further found that LPS promoted tumor growth (Fig. 5a) and STAT3 activation (Fig. 5b) in B16 ${ }^{\mathrm{NC}}$ tumors, but had less effect in B16 ${ }^{\text {STAT3 } \beta}$ tumors, indicating that STAT3 activation plays a positive role in TLR4 signalingmediated melanoma growth. IHC staining of tumor tissues showed that expression levels of Ki-67, CD31, $\mathrm{N}$-cadherin and vimentin were increased, while the expression of E-cadherin was decreased in LPS-stimulated $\mathrm{B} 16^{\mathrm{NC}}$ tumors, compared to that in $\mathrm{B} 16^{\mathrm{NC}}$ tumors without LPS stimulation (Fig. 5c). No significant differences were observed in Ki-67, CD31, E-cadherin, N-cadherin and vimentin expression in $\mathrm{B} 16^{\mathrm{STAT} 3} \beta$ tumors with and without LPS stimulation (Fig. 5c). The results of IHC staining imply an important role of STAT3 activation in TLR4 signaling-mediated melanoma growth, angiogenesis and EMT.

Activation of STAT3 has been shown to contribute to tumor-associated immunosuppression ${ }^{7}$. We found that LPS stimulation increased the percentage of splenic myeloid-derived suppressor cells (MDSCs; CD11b ${ }^{+} \mathrm{Gr}-1^{+}$ cells) in $\mathrm{B} 16^{\mathrm{NC}}$ tumor-bearing mice but not in $\mathrm{B} 16^{\mathrm{STAT} 3 \beta}$ tumor-bearing mice (Fig. 5d). MDSCs is responsible for immunosuppression that restricts the activity and tumor infiltration of cytotoxic CD8 $\mathrm{T}$ cells ${ }^{31}$. Percentages of splenic and tumor-infiltrating $\mathrm{CD} 8\left(\mathrm{CD}^{+} \mathrm{CD}^{+}\right) \mathrm{T}$ cells were significantly decreased in $\mathrm{B} 16^{\mathrm{NC}}$ tumor-bearing mice upon LPS stimulation (Fig. 5d). In B16 ${ }^{\mathrm{STAT} \beta}$ tumorbearing mice, LPS had a less potent decreasing effect on

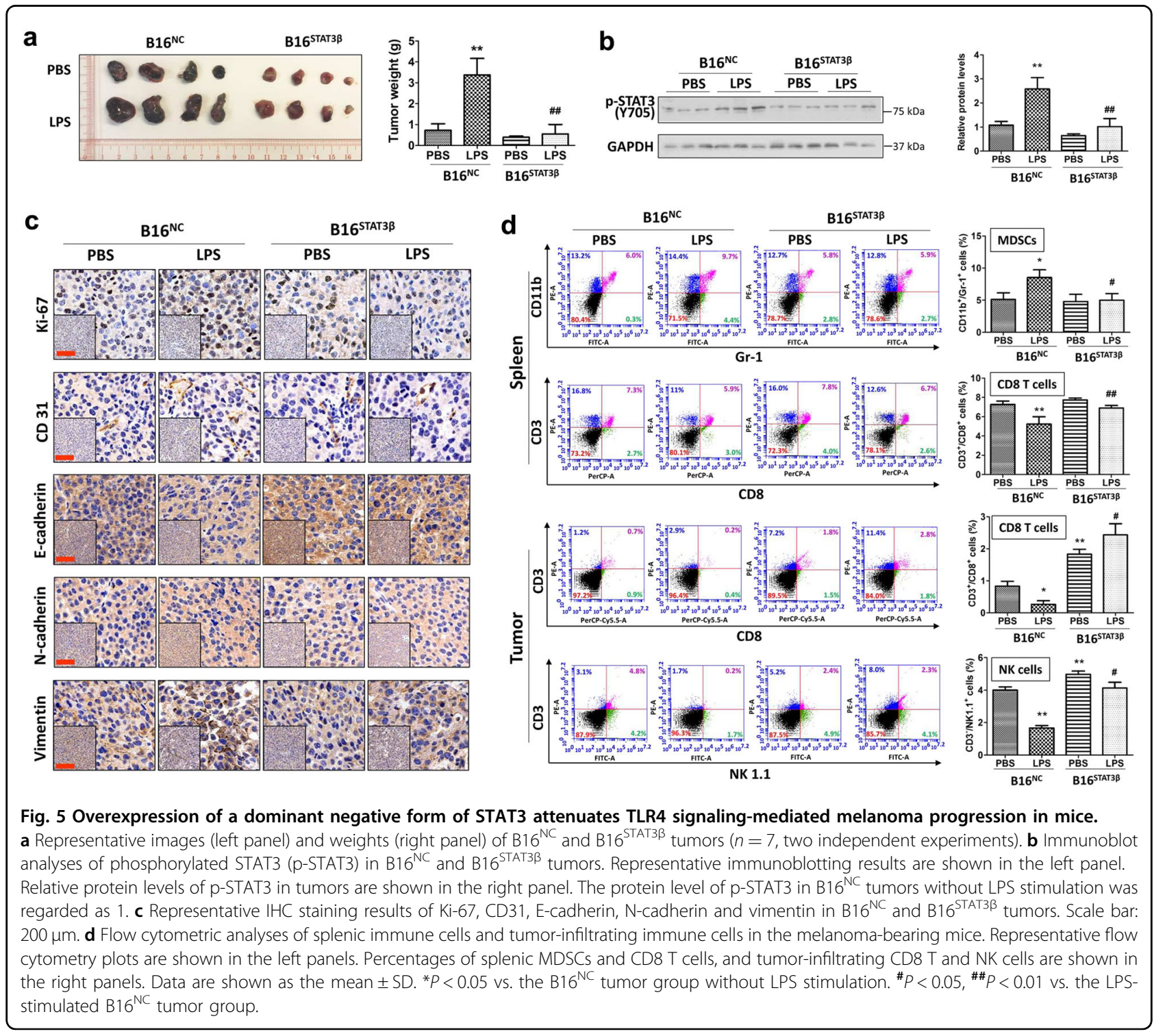


the number of splenic CD8 T cells than in $\mathrm{B} 16^{\mathrm{NC}}$ tumorbearing mice (Fig. $5 \mathrm{~d}$ ), suggesting a role of STAT3 activation in LPS-induced splenic CD8 $\mathrm{T}$ cell reduction. Of note, compared to PBS, LPS increased tumor-infiltrating CD8 $\mathrm{T}$ cells in B16 ${ }^{\text {STAT3 } \beta}$ tumor-bearing mice. This might be because LPS triggers TLR4/TRIF/type I interferon (IFN) signaling and subsequently upregulates $\mathrm{IFNs}^{32}$ to enhance CD8 T cell infiltration into tumors ${ }^{33}$. Like CD8 $\mathrm{T}$ cells, natural killer (NK) cells directly kill cancer cells ${ }^{34}$. Flow cytometric analyses showed that the number of NK $\left(\mathrm{NK} 1.1^{+} \mathrm{CD}^{-}\right)$cells was obviously decreased in LPSstimulated $\mathrm{B} 16^{\mathrm{NC}}$ tumors, but not in $\mathrm{B} 16^{\mathrm{NC}}$ tumors without LPS stimulation (Fig. 5d). Blocking STAT3 activation by expressing STAT3 $\beta$ in the tumors increased tumor-infiltrating NK cells and attenuated LPS-induced NK cell reduction (Fig. $5 \mathrm{~d}$ ). The changes of immune cell profiles imply that activation of STAT3 contributes to TLR4 signaling-mediated immunosuppression in melanoma microenvironment.

\section{Pharmacological inhibition of the TLR4/STAT3 pathway restrains melanoma cell proliferation in vitro and in vivo}

We have shown that STAT3 activation is important for TLR4 signaling-mediated melanoma progression. Next, we examined whether inhibiting the TLR4/STAT3 pathway suppresses proliferation of melanoma cells in vitro and in vivo. Parthenolide, a natural sesquiterpene lactone that inhibits TLR4 ${ }^{35}$ and STAT3 ${ }^{36}$ pathways in multiple cancer cells, and TAK-242, a TLR4 antagonist, were used here. Results showed that both parthenolide and TAK-242 dose-dependently lowered protein levels of TLR4, STAT3 and phosphorylated STAT3 (Fig. 6a) in and inhibited the proliferation (Fig. 6b) of A375 and B16 melanoma cells. To corroborate the in vitro data, we examined the effects of parthenolided on tumor growth in B16 melanoma-bearing mice. As shown in Fig. 6c-e, parthenolide dose-dependently decreased tumor weight and tumor volume, without affecting the body weight of mice. Immunoblotting data showed that parthenolide significantly lowered protein levels of TLR4, STAT3 and phosphorylated STAT3 in B16 tumors (Fig. 6f). These results suggest that inhibiting the TLR4/STAT3 pathway is a viable strategy for treating melanoma.

\section{Discussion}

In this study, our findings provide evidence that STAT3 is a key player in TLR4 signaling-mediated melanoma progression. TLR4 signaling activates STAT3 through MYD88 and TRIF, and subsequently upregulates a series of STAT3 target genes to promote melanoma progression (Fig. 7).

Studies have shown the contribution of TLR4 signaling in melanomagenesis. Takazawa et al. reported that LPS triggers TLR4/MYD88 signaling to promote migration of melanoma cells ${ }^{4}$. Bald et al. demonstrated that ultraviolet
(UV)-radiation induces TLR4/MYD88 axis-driven neutrophilic inflammation to promote melanoma angiogenesis and metastasis ${ }^{5}$. The study done by $\mathrm{Yu}$ et al. showed that LPS and a TLR4 endogenous ligand HMGB1 trigger TLR4 signaling to activate platelets, and thus promote melanoma metastasis ${ }^{6}$. In this study, we demonstrated that activation of TLR4/MYD88/STAT3 and TLR4/TRIF/ STAT3 pathways promotes proliferation of cultured melanoma cells. Moreover, in a mouse model, we demonstrated that STAT3 activation is required for TLR4 signaling-promoted melanoma progression in mice. We are interested in determining whether MYD88 and TRIF are involved in TLR4 signaling-mediated STAT3 activation in vivo in the future.

Receptor signaling can be constitutively activated by mutation(s) of the receptor and/or mutation(s) of molecule(s) in its signaling pathway(s) ${ }^{37}$. It is reported that there are $6.1 \%$ of patients with cutaneous melanoma harboring TLR4 non-synonymous mutations ${ }^{38}$. But the functions of the mutated TLR4 in melanoma are still unknown. No mutation of MYD88, TRIF and STAT3 genes has been reported in melanoma patients. Our paraffin-embedded melanoma samples, purchased from US Biomax, Inc., cannot be used to detect gene polymorphisms. We can collect additional patient melanoma samples to study the roles of gene polymorphisms of TLR4, MYD88, TRIF, and STAT3 in melanomagenesis in the future.

In the present study, we found that protein levels of TLR4 were significantly elevated in melanoma tissues compared to that in normal tissues. We also found that activation of STAT3 is a key event in TLR4 signalingmediated melanoma development. PU.1 has been reported to be responsible for TLR4 transcription ${ }^{39}$, and has been identified as a transcriptional target of STAT $3^{40}$. We therefore speculate that there is a TLR4/STAT3/PU.1 positive feedback loop in melanoma, which leads to the high expression of TLR4 and constitutive activation of STAT3. Further studies are required for establishing the TLR4/STAT3/PU.1 loop in melanoma.

In the tissue microarray analyses, we found that TLR4 expression and STAT3 phosphorylation are positively correlated in melanoma tissues. Although more than 90\% of cells detected in clinical melanoma specimens are cancer cells $^{41}$, there is a certain percentage of immune cells, such as T cells, DCs and macrophages, in the specimens. The TLR4 ligand LPS has been reported to activate STAT3 in $\mathrm{DCs}^{42}$ and macrophages ${ }^{43}$. Whether TLR4 expression is positively correlated with STAT3 activation in tumorinfiltrating immune cells is a question to be addressed.

It is known that, upon ligand binding, TLR4 recruits MYD88 and TRIF through Mal and TRAM, respectively, to activate downstream IKK/NF-kB, MAPK/AP1 and TKB1/IRF3 pathways ${ }^{44}$. In addition to MYD88 and TRIF, 


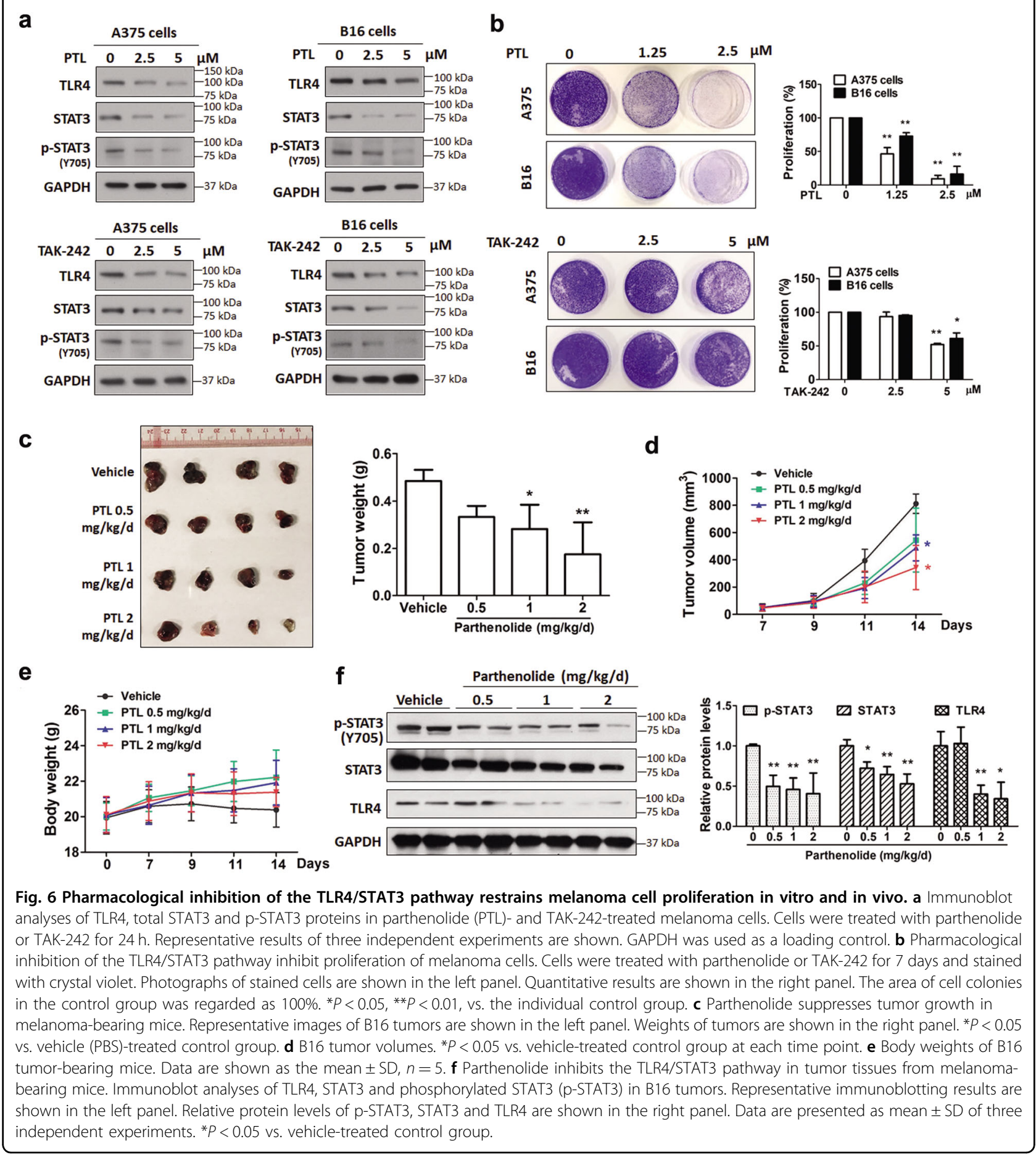

other molecules might be involved in the signaling cascades from TLR4 to STAT3 in melanoma. In pilot studies, we have found that inhibitors of IKK (BMS-345541), JNK (SP600125) and TLR4 (TAK-242), but not inhibitors of ERK (U0126) and p38 (SB203580), block LPS-induced STAT3 activation (Fig. S6), suggesting that IKK and JNK are involved in TLR4-signaling-mediated STAT3 activation in melanoma. To delineate the comprehensive molecular events involved in TLR4-mediated STAT3 activation, further studies are needed.

The TLR4/TRIF pathway, responsible for interferon production, is thought to promote innate immune responses ${ }^{45}$. MPLAs, a ligand that triggers TLR4/TRIF signaling ${ }^{14}$, has been used as an immunotherapy in 


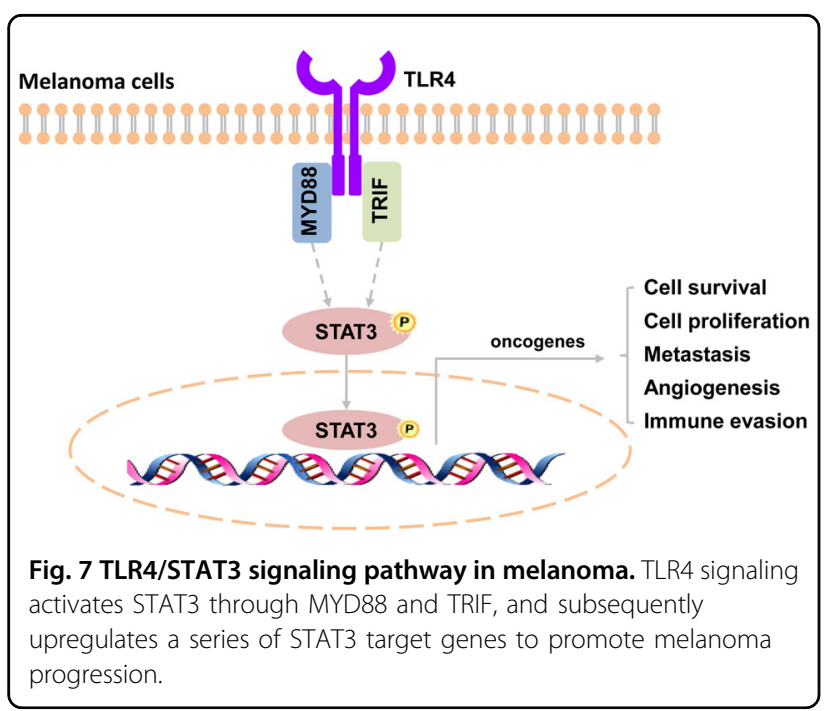

clinical trials for treating cancers including melanoma ${ }^{46}$. In this study, we showed that MPLAs activates STAT3 in melanoma cells, promotes melanoma cell proliferation, invasion and immunosuppressive cytokine secretion, and enhances pro-angiogenic effects of melanoma cells. Our findings suggest that caution should be taken when using MPLAs or other TLR4/TRIF pathway activators to treat melanoma. Combinational use of a TLR4/TRIF pathway activator and a STAT3 inhibiting agent may be a solution; however, further testing is required before clinical use.

STAT3 is a well-established target in experimental melanoma treatment ${ }^{7}$. STAT3 inhibitors that are being used in clinical trials exert anti-melanoma effects by inhibiting STAT3 activation ${ }^{47}$. However, their clinical efficacy is not ideal ${ }^{47}$. Up to now, no STAT3 inhibitor has been approved as a drug. Lowering STAT3 protein level may be able to more effectively block STAT3 signaling. Current strategies are to block STAT3 mRNA translation using decoy oligodeoxynucleotide (ODN) and antisense oligonucleotide (ASO) ${ }^{48}$. Unfortunately, physicochemical properties of ODN and ASO limit their clinical development ${ }^{48}$. In this study, we found that knockdown of TLR4 not only inhibits STAT3 activation, but also induces STAT3 protein degradation, suggesting an alternative approach to blocking STAT3 signaling in melanoma: genetically or pharmacologically inhibiting TLR4.

Parthenolide has anti-inflammatory and anticancer activities. It downregulates TLR4 expression in THP-1 monocytes ${ }^{49}$ and inhibits activation of NF-kB, a TLR4 downstream transcription factor, in melanoma cells ${ }^{50}$. In addition, parthenolide inhibits STAT3 activation and exerts anticancer effects in breast cancer, gastric cancer, prostate cancer and colon cancer cells ${ }^{36,51}$. Whether parthenolide affects both TLR4 and STAT3 in melanoma has not been reported. In this study, we showed that parthenolide, like the TLR4 antagonist TAK-242, lowers protein levels of TLR4, STAT3 and phosphorylated STAT3 in, and inhibits the proliferation of, melanoma cells. Parthenolide also downregulates protein levels of TLR4, STAT3 and phosphorylated STAT3 in tumor tissues and impedes tumor growth in melanoma-bearing mice. These findings indicate that targeting the TLR4/ STAT3 pathway is a viable strategy for treating melanoma.

In this study, we established TLR4/MYD88/STAT3 and TLR4/TRIF/STAT3 pathways in melanoma and demonstrated their pathogenic roles. TLR4 expression and constitutive STAT3 activation are also found in other cancers, such as liver ${ }^{52}$ lung $^{53,54}$, and stomach cancers ${ }^{55}$. In an attempt to extend our observations to other cancer types, we analyzed the correlation of TLR4 expression and STAT3 phosphorylation in human liver cancer, lung cancer and stomach cancer tissues using tissue microarray assays. In contrast to a previous report showing that TLR4 expression and STAT3 phosphorylation are positively correlated in primary tumors from liver cancer patients $(13 \text { cases })^{52}$, we found their correlation not significant in liver carcinoma tissues from the microarray (120 cases; BC03119b, US Biomax; Fig. S7A). These different results regarding the correlation between TLR4 expression and STAT3 activation in liver cancer may be because of the different sample sizes of the two analyses. Similarly, no significant correlation between TLR4 expression and STAT3 activation was observed in human lung cancer tissues (120 cases; BC041115d, US Biomax; Fig. S7B) and in stomach cancer tissues (102 cases; ST1021, US Biomax; Fig. S7C) from the microarrays. LPS has been shown to activate STAT3 signaling in cervical cancer ${ }^{56}$ and bladder cancer cells ${ }^{57}$. Whether TLR4 signaling activates STAT3 through the MYD88 and TRIF pathways in the two cancer types, as we observed in melanoma, and whether TLR4/ STAT3 signaling plays a pathogenic role in the two cancers warrant further investigations.

In conclusion, activation of STAT3 is a key event in TLR4 signaling-mediated melanoma progression. The TLR4/MYD88/STAT3 and TLR4/TRIF/STAT3 pathways established in this study shed novel insights into the pathophysiology of melanoma, and suggest new, potentially more effective, targets for treating melanoma.

\section{Acknowledgements}

This work was supported by grants GRF: 12125116, 12102918 and 12101519; JCYJ20160229210327924 and JCYJ20170817173608483;

GDNSF2016A030313007; NSFC: 81673649, 8187141799 and 81803788; HMRF: 14150571 and 15163441; and FRG2/17-18/032.

\section{Author details}

${ }^{1}$ Center for Cancer and Inflammation Research, School of Chinese Medicine, Hong Kong Baptist University, Hong Kong, China. ${ }^{2}$ Consun Chinese Medicines Research Centre for Renal Diseases, Hong Kong Baptist University, Hong Kong, China. ${ }^{3}$ Research and Development Centre for Natural Health Products, HKBU Shenzhen Research Institute and Continuing Education, Shenzhen, China. 
${ }^{4}$ JaneClare Transdermal TCM Therapy Laboratory, Hong Kong Baptist University, Hong Kong, China

\section{Author contributions}

X.Q.F. participated in experiment design, performed the experiments, analyzed the data and drafted the paper; B.L., participated in experiment design, data analysis and manuscript preparation; Y.P.W., and J.K.L., participated in performing the experiments and data analysis; K.W.T., participated in data analysis; P.L.Z., T.L., J.Y.C., C.L.Y., J.X.B., Y.X.L., and Y.J.C., participated in performing the experiments. Z.L.Y., conceived the study, designed experiments, supervised the project and finalized the paper.

\section{Conflict of interest}

The authors declare that they have no conflict of interest.

\section{Publisher's note}

Springer Nature remains neutral with regard to jurisdictional claims in published maps and institutional affiliations.

Supplementary Information accompanies this paper at (https://doi.org/ 10.1038/s41419-020-2440-1).

Received: 24 September 2019 Revised: 15 January 2020 Accepted: 16 January 2020

Published online: 20 April 2020

\section{References}

1. Testa, U., Castelli, G. \& Pelosi, E. Melanoma: genetic abnormalities, tumor progression, clonal evolution and tumor initiating cells. Med. Sci. (Basel) 5, 28 (2017).

2. Janssens, S. \& Beyaert, R. Role of Toll-like receptors in pathogen recognition. Clin. Microbiol. Rev. 16, 637-646 (2003).

3. Oblak, A. \& Jerala, R. Toll-like receptor 4 activation in cancer progression and therapy. Clin. Dev. Immunol. 2011, 609579 (2011).

4. Takazawa, Y. et al. Toll-like receptor 4 signaling promotes the migration of human melanoma cells. Tohoku. J. Exp. Med. 234, 57-65 (2014).

5. Bald, T. et al. Ultraviolet-radiation-induced inflammation promotes angiotropism and metastasis in melanoma. Nature 507, 109-113 (2014).

6. Yu, L. X. et al. Platelets promote tumour metastasis via interaction between TLR4 and tumour cell-released high-mobility group box1 protein. Nat. Commun. 5, 5256 (2014)

7. Kortylewski, M., Jove, R. \& Yu, H. Targeting STAT3 affects melanoma on multiple fronts. Cancer Metastasis Rev. 24, 315-327 (2005).

8. Zhou, L. et al. Significance of TLR4, MyD88 and STAT3 expression in colorectal cancer. Biomed. Res. 29, 317-322 (2018).

9. Li, T. et al. Inhibiting STAT3 signaling is involved in the anti-melanoma effects of a herbal formula comprising Sophorae Flos and Lonicerae Japonicae Flos. Sci. Rep. 7, 3097 (2017)

10. Cao, H. H. et al. Quercetin inhibits HGF/C-Met signaling and HGF-stimulated melanoma cell migration and invasion. Mol. Cancer 14, 103 (2015).

11. Cao, H. H. et al. Quercetin exerts anti-melanoma activities and inhibits STAT3 signaling. Biochem. Pharmacol. 87, 424-434 (2014).

12. Liu, Y. X. et al. A TCM formula comprising Sophorae Flos and Lonicerae Japonicae Flos alters compositions of immune cells and molecules of the STAT3 pathway in melanoma microenvironment. Pharmacol. Res. 142, 115-126 (2019).

13. Takeuchi, $\mathrm{O}$. et al. Differential roles of TLR2 and TLR4 in recognition of gramnegative and gram-positive bacterial cell wall components. Immunity 11, 443-451 (1999).

14. Goto, Y. et al. Activation of Toll-like receptors 2, 3, and 4 on human melanoma cells induces inflammatory factors. Mol. Cancer Ther. 7, 3642-3653 (2008).

15. Sun, H., Zhu, X., Cai, W. \& Qiu, L. Hypaphorine attenuates lipopolysaccharideinduced endothelial inflammation via regulation of TLR4 and PPAR- $\gamma$ dependent on PI3K/Akt/mTOR signal pathway. Int. J. Mol. Sci. 18, 844 (2017).

16. Shen, H., Tesar, B. M., Walker, W. E. \& Goldstein, D. R. Dual signaling of MyD88 and TRIF is critical for maximal TLR4-induced dendritic cell maturation. Immunol. 181, 1849-1858 (2008).
17. Mata-Haro, V. et al. The vaccine adjuvant monophosphoryl lipid A as a TRIFbiased agonist of TLR4. Science 316, 1628-1632 (2007).

18. Lee, E. F. et al. BCL-XL and $M C L-1$ are the key $B C L-2$ family proteins in melanoma cell survival. Cell Death Dis. 10, 342 (2019).

19. Hofmann, U. B., Westphal, J. R., Van Muijen, G. N. \& Ruiter, D. J. Matrix metalloproteinases in human melanoma. J. Invest. Dermatol. 115, 337-344 (2000).

20. Hamaï, A. et al. ICAM-1 has a critical role in the regulation of metastatic melanoma tumor susceptibility to CTL lysis by interfering with PI3KJAKT pathway. Cancer Res. 68, 9854-9864 (2008).

21. Ziani, L., Chouaib, S. \& Thiery, J. Alteration of the antitumor immune response by cancer-associated fibroblasts. Front. Immunol. 9, 414 (2018).

22. Rajabi, P. et al. The role of VEGF in melanoma progression. J. Res. Med. Sci. 17 534-539 (2012)

23. Ravindranathan, S. et al. Tumor-derived granulocyte colony-stimulating factor diminishes efficacy of breast tumor cell vaccines. Breast Cancer Res. 20, 126 (2018).

24. Zhu, C., Shen, H., Zhu, L., Zhao, F. \& Shu, Y. Plasminogen activator inhibitor 1 promotes immunosuppression in human non-small cell lung cancers by enhancing TGF-B1 expression in macrophage. Cell Physiol. Biochem. 44, 2201-2211 (2017).

25. Boettcher, S. et al. Endothelial cells translate pathogen signals into G-CSFdriven emergency granulopoiesis. Blood 124, 1393-1403 (2014).

26. Rébé, C., Végran, F., Berger, H. \& Ghiringhelli, F. STAT3 activation: a key factor in tumor immunoescape. JAKSTAT 2, e23010 (2013).

27. Carpenter, R. L. \& LO, H. W. STAT3 target genes relevant to human cancers. Cancers (Basel) 6, 897-925 (2014).

28. Lin, $X$. et al. PAI-1/PIAS3/Stat3/miR-34a forms a positive feedback loop to promote EMT-mediated metastasis through Stat3 signaling in non-small cell lung cancer. Biochem. Biophys. Res. Commun. 493, 1464-1470 (2017).

29. Caldenhoven, E. et al. STAT3beta, a splice variant of transcription factor STAT3, is a dominant negative regulator of transcription. J. Biol. Chem. 271, 13221-13227 (1996).

30. Wu, Y. \& Zhou, B. P. New insights of epithelial-mesenchymal transition in cancer metastasis. Acta Biochim. Biophys. Sin. (Shanghai) 40, 643-650 (2008).

31. Marvel, D. \& Gabrilovich, D. I. Myeloid-derived suppressor cells in the tumor microenvironment: expect the unexpected. J. Clin. Invest. 125, 3356-3364 (2015).

32. Richez, C. et al. TLR4 ligands induce IFN-alpha production by mouse conventional dendritic cells and human monocytes after IFN-beta priming. J. Immunol. 182, 820-828 (2009).

33. Oelkrug, C. \& Ramage, J. M. Enhancement of T cell recruitment and infiltration into tumours. Clin. Exp. Immunol. 178, 1-178 (2014).

34. Wu, J. \& Lanier, L. L. Natural killer cells and cancer. Adv. Cancer Res. 90, 127-156 (2003).

35. Sohma, I. et al. Parthenolide, an NF-kB inhibitor, suppresses tumor growth and enhances response to chemotherapy in gastric cancer. Cancer Genomics Proteom. 8, 39-47 (2011).

36. Li, H., Lu, H., Lv, M., Wang, Q. \& Sun, Y. Parthenolide facilitates apoptosis and reverses drug-resistance of human gastric carcinoma cells by inhibiting the STAT3 signaling pathway. Oncol. Lett. 15, 3572-3579 (2018).

37. Scaltriti, M. \& Baselga, J. The epidermal growth factor receptor pathway: a model for targeted therapy. Clin. Cancer Res. 12, 5268-5272 (2006).

38. Fels Elliott, D. R. et al. Impact of mutations in Toll-like receptor pathway genes on esophageal carcinogenesis. PLoS Genet. 13, e1006808 (2017).

39. Lichtinger, M., Ingram, R., Hornef, M., Bonifer, C. \& Rehli, M. Transcription factor PU.1 controls transcription start site positioning and alternative TLR4 promoter usage. J. Biol. Chem. 282, 26874-26883 (2007).

40. Hegde, S. et al. Stat3 promotes the development of erythroleukemia by inducing Pu.1 expression and inhibiting erythroid differentiation. Oncogene $\mathbf{2 8}$ 3349-3359 (2009).

41. Mengus, C. et al. In vitro modeling of tumor-immune system interaction. ACS Biomater. 4, 314-323 (2018).

42. Kortylewski, M. et al. Toll-like receptor 9 activation of signal transducer and activator of transcription 3 constrains its agonist-based immunotherapy. Cancer Res. 69, 2497-2505 (2009).

43. Chaves de Souza, J. A. et al. SOCS3 expression correlates with severity of inflammation, expression of proinflammatory cytokines, and activation of STAT3 and p38 MAPK in LPS-induced inflammation in vivo. Mediators Inflamm. 2013, 650812 (2013). 
44. Kawasaki, T. \& Kawai, T. Toll-like receptor signaling pathways. Front. Immunol. 5, 461 (2014).

45. Li, K., Qu, S., Chen, X., Wu, Q. \& Shi, M. Promising targets for cancer immunotherapy: TLRs, RLRs, and STING-mediated innate immune pathways. Int. J. Mol. Sci. 18, 404 (2017).

46. Cluff, C. W. Monophosphoryl lipid A (MPL) as an adjuvant for anti-cancer vaccines: clinical results. Adv. Exp. Med. Biol. 667, 111-123 (2010).

47. Bharadwaj, U., Kasembeli, M.M. \& Tweardy, D.J. STAT Inhibitors in Cancer. Cancer Drug Discov. Dev. https://doi.org/10.1007/978-3-319-42949-6_5 (2016).

48. Yu, C.L., et al. STAT inhibitors in cancer. Cancer drug discovery and development. Historical development of STAT3 inhibitors and early results in clinical trials, https://doi.org/10.1007/978-3-319-42949-6_4 (2016).

49. Li, S. et al. Parthenolide inhibits LPS-induced inflammatory cytokines through the toll-like receptor 4 signal pathway in THP-1. cells Acta Biochim. Biophys. Sin. (Shanghai) 47, 368-375 (2015).

50. Czyz, M., Lesiak-Mieczkowska, K. Koprowska, K., Szulawska-Mroczek, A. \& Wozniak, M. Cell context-dependent activities of parthenolide in primary and metastatic melanoma cells. Br. J. Pharm. 160, 1144-1157 (2010).
51. Liu, M. et al. Parthenolide inhibits STAT3 signaling by covalently targeting janus kinases. Molecules 23, 1478 (2018).

52. Lin, A. et al. TLR4 signaling promotes a COX-2/PGE2/STAT3 positive feedback loop in hepatocellular carcinoma (HCC) cells. Oncoimmunology 5, e1074376 (2015).

53. Wang, $K$ et al. Expression of TLR4 in non-small cell lung cancer is associated with PD-L1 and poor prognosis in patients receiving pulmonectomy. Front. Immunol. 8, 456 (2017).

54. Chen, $X$. et al. Circular RNA circHIPK3 modulates autophagy via MIR124-3pSTAT3-PRKAAAMPKa signaling in STK11 mutant lung cancer. Autophagy $\mathbf{2 8}$ 1-13 (2019).

55. Zhou, $Y$. et al. Induction of pro-inflammatory response via activated macrophage-mediated NF-KB and STAT3 pathways in gastric cancer cells. Cell Physiol. Biochem. 47, 1399-1410 (2018).

56. Ma, L., Feng, L., Ding, X. \& Li, Y. Effect of TLR4 on the growth of SiHa human cervical cancer cells via the MyD88-TRAF6-TAK1 and NF-KB-cyclin D1-STAT3 signaling pathways. Oncol. Lett. 15, 3965-3970 (2018).

57. Ying, $H$. et al. TLR4 mediates MAPK-STAT3 axis activation in bladder epithelial cells. Inflammation 36, 1064-1074 (2013). 